\title{
Sheet level sets and the representation theorem for intuitionistic fuzzy sets
}

\section{Gökhan Çuvalcıŏglu, Sinem (Yılmaz) Tarsuslu and Emine Demirbaş}

\author{
Department of Mathematics, Mersin University \\ 33016, Mersin Turkey \\ e-mails: gcuvalcioglu@gmail.com, s i nemnyilmaz@gmail.com, \\ eminesdemirbas@gmail.com
}

Received: 20 March 2019

Revised: 15 April 2019

Accepted: 24 April 2019

\begin{abstract}
In this study, the representation theorem and a partition for intuitionistic fuzzy sets were given. For those properties, intuitionistic fuzzy sheet $t$-level set, intuitionistic fuzzy $\alpha-t$ block level set and intuitionistic fuzzy $t$-subset were used.
\end{abstract}

Keywords: Intuitionistic Fuzzy sets, Sheet $t$-level, IFS $\alpha$ - $t$ block level, Intuitionistic Fuzzy $t$-Subset, Representation theorem.

2010 Mathematics Subject Classification: 03E72, 03E99.

\section{Introduction}

Fuzzy sets were introduced by Zadeh [10] as an extension of classical (crisp) sets by expanding the value of membership of an element in any universal set to the real unit interval $[0,1]$. If we choose $X$ as a universe set, then the function $\mu: X \rightarrow[0,1]$ is called a fuzzy set over $X$. The family of fuzzy sets is denoted by $F S(X)$, for brevity. For $x \in X, \mu(x)$ is called the membership degree of $x$ and $1-\mu(x)$ is called the non-membership degree of $x$.

One of the extension of the fuzzy sets is introduced by Atanassov in 1983 [1]. These sets were defined from an universe set to the lattice $[0,1] \times[0,1]$, and called intuitionistic fuzzy sets. The family of intuitionistic fuzzy sets is denoted by $\operatorname{IFS}(X)$, for brevity. In any intuitionistic fuzzy set, any element of universe has membership, non-membership and hesitation degrees, the sum of which is 1 . 
For fuzzy sets, the concept of level sets was originally introduced by Zadeh [1]. By level sets, we are able to descend to the classical sets and get some properties for the fuzzy sets using classical set properties. This method can allow us to extend the operations and structures defined on crisp sets to the case of fuzzy sets [3].

Atanassov carried the idea of level sets of fuzzy sets to intuitionistic fuzzy sets [2]. The definition of the level set of any intuitionistic fuzzy set is also a crisp set, like in the fuzzy sets case $[5,7,8]$.

The different type of level set of intuitionistic fuzzy sets was given by author [4]. In contrast to the other definition, level sets were defined as $s$-fuzzy sets. In this way, one can say that a level set of any intuitionistic fuzzy set need not be a classical set, but the level of it is classical set.

By means of the definitions of intuitionistic fuzzy sheet $t$-level set, intuitionistic fuzzy $\alpha-t$ block level set and intuitionistic fuzzy $t$-subset of the representation theorem can be stated.

\section{Intuitionistic fuzzy sheet and block level sets}

In this section, for our purposes, we will give some definitions and properties of the intuitionistic fuzzy sets with respect to the level sets. The first definition of a level set for IFSs is the following.

Definition 1: [2] Let $A \in \operatorname{IFS}(X)$. Then the $(\alpha, \beta)$-level of $A$ and the strong $(\alpha, \beta)$-level of $A$ are crisp subsets of the $X$, respectively, and are given by

$$
\begin{gathered}
C_{(\alpha, \beta)}(A)=\left\{x \mid x \in X \text { such that } \mu_{A}(x) \geq \alpha, v_{A}(x) \leq \beta\right\} \\
C_{<\alpha, \beta>}(A)=\left\{x \mid x \in X \text { such that } \mu_{A}(x)>\alpha, v_{A}(x)<\beta\right\}
\end{gathered}
$$

where $\alpha, \beta \in[0,1]$ with $\alpha+\beta \leq 1$.

Definition 2: [4] Let $X$ be a set and $A=\left\{\left\langle x, \mu_{A}(x), v_{A}(x)\right\rangle \mid x \in X\right\} \in \operatorname{IFS}(X)$. If $t \in[0,1]$ then sheet $t$-level of $A$ is defined as following

$$
A(t)=\left\{<x, \mu_{A}(x), v_{A}(x)>\mid \mu_{A}(x)+v_{A}(x)=t, x \in X\right\} .
$$

The sheet $t$-level of an intuitionistic fuzzy set, for each $t \in[0,1]$, indicates discrete sheets. Each sheet $t$-level set can be specified as a fuzzy set. From the above definition, it is easily seen that for every $t \in[0,1], A(t) \in F S(X)$. Because $A(t): X \rightarrow[0, t]$ and $[0, t] \sim[0,1]$. For short notation, $A(t)$ will be called $s$-fuzzy set on $X$, i.e. $\left.A(t) \in F S_{S}(X)\right)$.

Proposition 1: [4] Let $X$ be a set and $A, B \in \operatorname{IFS}(X)$. For every $t \in[0,1]$,

1. $(A \sqcup B)(t)=A(t) \sqcup B(t)$

2. $A(t) \sqcap B(t)=(A \sqcap B)(t)$

3. $\left(A^{c}(t)\right)^{c}=A(t)$

Proposition 2: [4] Let $X$ be a set and $A \in \operatorname{IFS}(X)$. If $t, s \in[0,1]$, then either $A(t) \sqcap A(s)=\varnothing$ or $t=s$.

Corollary 2: [4] There exist an equivalence relation on $X$ such that the sheet $t$-levels are equivalence class of that relation.

Definition 3: [4] Let $X$ be a set and $A \in \operatorname{IFS}(X)$. If $t \in[0,1]$ and $\alpha \in[0, \mathrm{t}]$, then 


$$
A(t)_{\alpha}=\{x \mid x \in X, A(t)(x) \geq(\alpha, t-\alpha)\}
$$

is called $\alpha-t$ block level of $A$.

From above definition, it is seen easily that $A(t)_{\alpha}$ is a crisp set. Firstly, we obtained a $s$-fuzzy set by $A(t)$ and then it is reduced to the $A(t)_{\alpha}$ crisp set. Some special cases are as follows.

Proposition 3: [4] Let $X$ be a set and $A \in \operatorname{IFS}(X)$. If $t \in[0,1]$ then

$$
A(t)_{t}=\left\{x \mid x \in X, \mu_{A}(x)=t \wedge v_{A}(x)=0\right\} .
$$

Difference among the different definition of level sets for intuitionistic fuzzy sets, we can examine the following examples.

Example 1: Let $X=\{a, b, c, d, e\}$ and

$$
A=\{(a, 0.5,0.4),(b, 0.2,0.3),(c, 0.5,0.3),(d, 0.4,0.4),(e, 0.4,0.1)\}
$$

$A(0.5)_{(0.3)}=\{e\}$ but $C_{(0.3,0.5)}(A)=\{a, c, d, e\}$ and $C_{(0.5,0.3)}(A)=\{c\}$.

$A(0.8)_{(0.5)}=\{c\}$ but $0.8+0.5>1$ so, we cannot obtain $C_{(0.8,0.5)}(A)$ or $C_{(0.5,0.8)}(A)$.

Example 2: Let $X=\{a, b, c, d, e\}$ and

$$
A=\{(a, 0.3,0.3),(b, 0.3,0.1),(c, 0.2,0.2),(d, 0.1,0.5),(e, 0.4,0.2)\}
$$

$A(0.6)_{(0.2)}=\{a, e\}$ but $C_{(0.6,0.2)}(A)=\varnothing$ and $C_{(0.2,0.6)}(A)=X$.

Example 3: Let $X=\{a, b, c, d, e\}$ and

$$
A=\{(a, 0.1,0.2),(b, 0.4,0.3),(c, 0.6,0.2),(d, 0.7,0.1),(e, 0.2,0.5)\}
$$

$A(0.3)_{(0.2)}=\varnothing$ but $C_{(0.2,0.3)}(A)=\{b, c, d\}$ and $C_{(0.3,0.2)}(A)=\{c, d\}$.

From the examples, $(\alpha, \beta)$-level of an intuitionistic fuzzy set $A$ and $\alpha-t$ block level of $A$ are different sets. For all $t \in[0,1]$ and $\alpha \in[0, \mathrm{t}]$, we can determine $\alpha$-t block level of $A$, if $\alpha+t>1$ then we cannot determine $(\alpha, \beta)$-level of $A$. Consequently, $\alpha-t$ block level of an intuitionistic fuzzy set allows a more extensive studying area.

Proposition 4: [4] Let $X$ be a set and $A \in \operatorname{IFS}(X)$. If $t \in[0,1]$ and $\alpha, \beta \in[0, t]$ such that $\alpha \leq \beta$, then

$$
A(t)_{\beta} \subseteq A(t)_{\alpha}
$$

Proposition 5: [4] Let $X$ be a set and $A, B \in \operatorname{IFS}(X)$. If $t \in[0,1]$ and $\alpha \in[0, t]$, then

1. $A(t)_{\alpha} \cup B(t)_{\alpha}=(A(t) \sqcup B(t))_{\alpha}$

2. $A(t)_{\alpha} \cap B(t)_{\alpha}=(A(t) \sqcap B(t))_{\alpha}$

3. $A(t)_{\alpha}{ }^{c}=t-A(t)_{\alpha},(t(x)=t)$

Theorem 1: [4] Let $X$ be a set and $A \in \operatorname{IFS}(X)$. For $t, s \in[0,1]$ and $\alpha \in[0, t], \beta \in[0, s]$ if $\alpha \leq \mathrm{s}$, then

1. $A(t)_{\alpha} \cup A(t)_{\beta}=A(t)_{\min (\alpha, \beta)}$

2. $A(t)_{\alpha} \cap A(t)_{\beta}=A(t)_{\max (\alpha, \beta)}$

Proposition 6: [4] Let $X$ be a set and $A \in \operatorname{IFS}(X)$. If $t \in[0,1]$ and $\alpha \in[0, t]$ then $A(t)_{\alpha} \subset A(t)_{t-\alpha}$ or $A(t)_{t-\alpha} \subset A(t)_{\alpha}$.

Definition 4: [4] Let $X$ be a set and $A \in \operatorname{IFS}(X)$. If $t \in[0,1]$ then 


$$
A^{t}=\left\{<x, \mu_{A}(x), v_{A}(x)>\mid<x, \mu_{A}(x), v_{A}(x)>\in A(r), \forall t \leq r\right\}
$$

is called $t$-subset of $A$.

From the definition, it can be easily said that every $t$-subset of an intuitionistic fuzzy sets again an intuitionistic fuzzy subset. Let us examine the Example below:

Example 4: Let $X=\{a, b, c, d, e\}$ and

$$
A=\{(a, 0.1,0.2),(b, 0.4,0.3),(c, 0.6,0.2),(d, 0.7,0.1),(e, 0.2,0.5)\}
$$

then for $t=0.7$,

$$
A^{t}=\{(b, 0.4,0.3),(c, 0.6,0.2),(d, 0.7,0.1),(e, 0.2,0.5)\}
$$

and for $t \leq 0.3, A^{t}=A$.

Remark: [4] In fuzzy set theory union and intersection of two level sets is again a level set of the fuzzy set. But this is not true for block $t$-level sets of an IFS.

\section{The Representation Theorem for IFSs}

The representation theorem for fuzzy sets was studied by several authors [6,9]. But all of them using the sub structure for the representation. For intuitionistic fuzzy sets, it can be given using same idea with block $t$-levels and intuitionistic fuzzy $t$-sets.

Theorem 2: Let $X$ be universe, $\left\{X_{t}\right\}_{t \in I}$ a family of FS. Then $\exists A \in \operatorname{IFS}(X)$ such that $A^{t}=X_{t} \Leftrightarrow$

$$
\begin{aligned}
& \text { i. } t \leq s \Rightarrow X_{t} \supseteq X_{s} \\
& \text { ii. } t_{1} \leq t_{2} \leq \cdots, t_{n} \rightarrow t \Rightarrow \bigcap_{n=1}^{\infty} X_{t_{n}}=X_{t}
\end{aligned}
$$

Proof: The first statement is clearly obtained from the definition of $A$.

Now, the conditions (i) and (ii) satisfied. We define a set $A: X \rightarrow I \times I$ such that for $x \in X$,

$$
A(x)=\sup \left\{t \mid<x, \mu_{A}(x), \mu_{A}(x)>\in X_{t}\right\} .
$$

From the definition of $A$,

$a \in X_{r} \Rightarrow r \in\left\{t \mid a \in X_{t}\right\} \Rightarrow \sup \left\{t \mid<a, \mu_{A}(a), \mu_{A}(a)>\in X_{t}\right\} \geq r \Rightarrow A(a) \geq r \Rightarrow a \in A^{r}$

$a \in A^{r} \Rightarrow \sup \left\{t \mid<a, \mu_{A}(a), \mu_{A}(a)>\in X_{t}\right\} \geq r \Rightarrow A(a) \geq r$

If $A(a)>r$, then $\exists r_{1} \in I$ such that $a \in X_{r_{1}}$ and $r_{1}>r$. Therefore, $X_{r_{1}} \subseteq X_{r}$, so, $a \in X_{r}$.

If $A(a)=r$, then $\sup \left\{t \mid<a, \mu_{A}(a), \mu_{A}(a)>\in X_{t}\right\}=r$. Therefore, $\exists r_{n} \in I$ such that $r_{n} \uparrow r$. From the hypothesis, $\bigcap_{\mathrm{n}=1}^{\infty} X_{r_{n}}=X_{r}$. From this equality we get $a \in X_{r}$.

Corollary 2: There exist a family of $s$-fuzzy sets such that it is a partition of any intuitionistic fuzzy set.

Proof: Let $A \in I F S(X)$, then from the above theorem there exists a family $\left\{X_{t}: t \in I\right\}$ such that the elements of it are $t$-subsets of $A$. From the definition of $A(t)$ sheet $t$-levels, we have $A(t) \cap \bigcup_{t \neq s} A_{s}=\varnothing$ and $A^{t}(k)=A(k)$, therefore $A^{t}=\bigcup_{k \geq t} A(k)$. 


\section{Conclusion}

The essence of the representation theorem has to do with the possibility of constructing an intuitionistic fuzzy set from a family of special type of IFSs and $s$-fuzzy sets. Also, it was proved that all intuitionistic fuzzy sets have a partition which includes $s$-fuzzy sets. By means of this property, equivalence relations of intuitionistic fuzzy sets can be studied. As a result, partition trees can be studied. Taking into account the new different type levels definitions, we can ask ourselves whether the levels of an intuitionistic fuzzy set are either a group or not.

\section{Acknowledgements}

This paper is supported by Mersin University, Scientific Research Projects with code 2017-2AP2-2616.

\section{References}

[1] Atanassov K. T. (1983). Intuitionistic Fuzzy Sets, VII ITKR's Session, Sofia, June 1983 (Deposed in Centr. Sci.-Techn. Library of the Bulg. Acad. of Sci., 1697/84, in Bulgarian). Reprinted: Int. J. Bioautomation, 2016, 20 (S1), S1-S6.

[2] Atanassov K. T. (1999). Intuitionistic Fuzzy Sets: Theory and Applications, Heidelberg, Springer.

[3] Çuvalcıoğlu, G., \& Yılmaz, S. (2014). Some properties of intuitionistic fuzzy equivalence relations and class trees w.r.t. intuitionistic fuzzy equivalence relations, Adv. Studies Contemp. Math., 24 (4), 77-86.

[4] Çuvalcıoğlu, G. \& Tarsuslu (Yılmaz), S. The block and sheet levels of intuitionistic fuzzy sets, Journal of Universal Mathematics, (under review).

[5] Maheswari, A., Karthikeyan S. \& Palanivelrajan, M. (2012). Characterization of level sets of intuitionistic L-fuzzy semi filter of lattices, Notes on Intuitionistic Fuzzy Sets, 18 (2), $21-25$.

[6] Negoita, C. V., \& Ralescu, D. A. (1974). Multimi Vagi Si Aplicatiile Lor, Ed. Technica, Bucharest.

[7] Saravanan, V., \& Sivakumar D. (2012). Level subsets of intuitionistic fuzzy sub-semiring of a semiring, International Journal of Fuzzy Mathematics and Systems, 2 (4), 423-432.

[8] Sharma, P. K. (2011). ( $\alpha, \beta)$-level of intuitionistic fuzzy groups, International Mathematical Forum, 53 (6), 2605-2614.

[9] Yager, R. R. (2010). Level sets and the representation theorem for intuitionistic fuzzy sets, Soft Computing, 14, 1-7.

[10] Zadeh, L. A. (1965). Fuzzy sets, Information and Control, 8, 338-353. 